\title{
Use of Network Analysis to Establish Neurosurgical Parameters in Gliomas and Epilepsy
}

\author{
Satoshi MAESAWA, ${ }^{1,2}$ Epifanio BAGARINAO, ${ }^{1}$ Masazumi FuJII, ${ }^{3}$ \\ Miyako FUTAMURA, ${ }^{4}$ and Toshihiko WAKABAYASHI ${ }^{2}$ \\ ${ }^{1}$ Brain and Mind Research Center, Nagoya University, Nagoya, Aichi; \\ ${ }^{2}$ Department of Neurosurgery, Nagoya University Graduate School of Medicine, \\ Nagoya, Aichi; \\ Department of Neurosurgery ${ }^{3}$ and Rehabilitation Center, ${ }^{4}$ Fukushima Medical University, \\ Fukushima, Fukushima
}

\begin{abstract}
Cutting-edge neuroimaging technologies can facilitate preoperative evaluation in various neurosurgical settings. Surgery for gliomas and epilepsy requires precise localization for resection due to the need to preserve (or perhaps improve) higher cognitive functions. Accordingly, a hodological approach should be taken that considers subcortical networks as well as cortical functions within various functional domains. Resting state functional magnetic resonance imaging (fMRI) has the potential to provide new insights that are valuable for this approach. In this review, we describe recent developments in network analysis using resting state fMRI related to factors in glioma and epilepsy surgery: the identification of functionally dominant areas, evaluation of cognitive function by alteration of resting state networks (RSNs), glioma grading, and epileptic focus detection. One particular challenge that is close to realization is using fMRI for the identification of sensorimotor- and language-dominant areas during a task-free resting state. Various RSNs representative of the default mode network demonstrated at least some alterations in both patient groups, which correlated with behavioral changes including cognition, memory, and attention, and the development of psychosis. Still challenging is the detection of epileptic foci and propagation pathways when using only network analysis with resting state fMRI; however, a combined method with simultaneous electroencephalography has produced promising results. Consequently, network analysis is expected to continue to advance as neuroimaging technology improves in the next decade, and preoperative evaluation for neurosurgical parameters through these techniques should improve parallel with them.
\end{abstract}

Key words: resting state functional magnetic resonance imaging (fMRI), resting state networks, electroencephalography-fMRI, gliomas, epilepsy

\section{Introduction}

Recent advances in neuroimaging have made it feasible to examine human brain connectivity systematically. In 2009, the National Institutes of Health announced the opening of the Human Connectome Project (HCP), whose principal objective is understanding the totality and variability of human brain connectivity. ${ }^{1)}$ We believe that new findings regarding neural networks, possibly obtained in the HCP, could be utilized for determining various neurosurgical parameters, especially for glioma and epilepsy surgeries. In fact, using the same leading-edge imaging technologies as those

Received November 28, 2015; Accepted January 19, 2016 used for the HCP will facilitate the upgrade of preoperative neurosurgical evaluations. In glioma surgery, a positive outcome requires resecting the maximum amount of tumoral tissue even when it has invaded the normal parenchyma, while preserving as much of the normal adjacent tissue and its cerebral functions, including higher cognition. In epilepsy surgery, precise identification of the epileptic focus and delineation of the resectable areas are required, while again preserving or even improving current neurological function. For these purposes, both cortical topology and subcortical networks must be interpreted for the individual patient. This concept, which considers the neural pathways that include the subcortical networks, was recently termed the "hodological approach.", 
Recently, among novel imaging technologies, there has been an increase in interest in the application of resting state functional magnetic resonance imaging (fMRI). This technique investigates synchronous activities between distinct regions during the taskfree resting state, and identifies various resting state networks (RSNs). The analysis of RSNs could contribute to a hodologically based preoperative interpretation of neural networks. This technique is relatively new. Although acquisition of images is sufficiently simple, the analysis and interpretation of the results are relatively complicated and requires expertise. This has limited the utility of the technique to certain neuroimaging centers so far. However, the technology has begun to improve in terms of user-friendliness, which should lead to a much more broad usage base fairly quickly.

In this review, we discuss the studies of RSNs performed by resting state fMRI, and present the potential clinical applications focusing on glioma and epilepsy. Furthermore, a technological advance coupling resting state fMRI with simultaneous electroencephalography (EEG-fMRI) has been recently utilized for epilepsy, demonstrating strong potential regarding focus detection and network analysis worthy of discussion here.

\section{Fundamentals of Resting State fMRI}

fMRI using various tasks such as finger tapping or verb generation is one of the most important methods for preoperative functional mapping in the brain in patients with gliomas or epilepsy. However, its sensitivity is limited by a poor signal:noise ratio, the reliability of the tasks, and the participant's performance. A meta-analysis of 63 published studies found that task-based fMRI has less than $50 \%$ inter-individual reproducibility, in even healthy subjects. ${ }^{3)}$ Task-based fMRI is particularly challenging for patients with hemiparesis, cognition, or attention deficits. Thus, resting state fMRI could reduce false negative or false positive errors associated with task-performance in the presurgical identification of functionally localized areas.

Resting state fMRI focuses on spontaneous, low frequency fluctuations of the time course of the blood oxygen level-dependent (BOLD) signal whose frequencies are commonly less than $0.1 \mathrm{~Hz}$. The significance of this method was firstly reported by Biswal et al. in 1995, who showed that the time course of the BOLD signal in the unilateral sensory cortex identified by standard task-based fMRI correlated strongly with the homologous area of the contralateral hemisphere. ${ }^{4)}$ A variety of RSNs characterized by synchronized spontaneous low frequency fluctuations have been identified since then. The most fundamental RSN has been dubbed the default mode network (DMN), which was initially identified with PET scans by Raichle et al. ${ }^{5)}$ They found a consistent set of regions that were active during the resting state, but inactive during task performance. A few years later, the DMN was identified with resting state fMRI by Greicius et al., ${ }^{6)}$ and its components have been verified in many studies. The DMN, which includes the posterior cingulate cortex, the ventromedial prefrontal cortex (VMPFC), the angular gyrus, and the medial temporal lobe, is the most studied network that can be readily detected in resting state fMRI. It is highly reproducible across both imaging sessions and different subjects. ${ }^{7}$ Its precise function is still largely unknown, but the individual regions comprising it are hypothesized to be involved in stimulus-independent thoughts,$^{8)}$ introspection, ${ }^{9)}$ and the integration of autobiographical, self-monitoring, and related social cognitive functions. ${ }^{10)}$ Alterations of the default mode network and/or its functional connectivity have been reported in several neurological conditions, including Alzheimer's disease, autism, and schizophrenia. ${ }^{11-14)}$

Several other RSNs have been identified, including a somatosensory network, ${ }^{4)}$ a visual network, ${ }^{15,16)}$ an auditory network, ${ }^{17)}$ a language network, ${ }^{18)}$ both dorsal and ventral attention networks, ${ }^{19)}$ an executive control network (ECN), ${ }^{20,21)}$ and a salience network $^{22)}$ (Table 1). These networks were termed as "task-positive" or "extrinsic," whereas the DMN was termed as "task-negative" or "intrinsic." The analysis methods for RSNs are varied. Seed-based analysis is a fundamental approach applied in numerous studies. In it, a region of interest (ROI) is selected, which can create possible error via selection bias and a lack of objectivity in the choice of ROI. Another popular approach is independent component analysis (ICA), a mathematical technique that maximizes independence among its components statistically. ${ }^{15)}$ This technique does not require the a priori selection of an ROI, and enables analysts to distinguish each RSN automatically (Fig. 1). However, it requires the initial setup of a number of components, as well as clear discrimination of important components from noise.

The electrophysiological basis of RSNs, obtained by analysis of low frequency fluctuations of BOLD signals in resting state fMRI, has not been comprehensively understood. Each region of the RSN (i.e., sensorimotor network) demonstrates high concordance with activated or deactivated areas in fMRI with relevant tasks (i.e., finger tapping). Accordingly, RSNs are able to substantiate particular brain functions. 
Table 1 The main resting state networks of the human brain

\begin{tabular}{|c|c|c|}
\hline Resting state networks & Anatomical locations & Publications \\
\hline Default mode network & $\begin{array}{l}\text { the posterior cingulate cortex, the ventromedial prefrontal } \\
\text { cortex, the angular gyrus, and the medial temporal lobe }\end{array}$ & $\begin{array}{l}\text { Raichle et al., 2001,5) } \\
\text { Greicius et al., 20036) }\end{array}$ \\
\hline Dorsal attention network & $\begin{array}{l}\text { the intraparietal sulcus, the junction of the precentral, and } \\
\text { the superior frontal sulcus (frontal eye field) }\end{array}$ & Fox et al., 2006 ${ }^{19)}$ \\
\hline Ventral attention network & $\begin{array}{l}\text { the temporo-parietal junction, the ventral frontal cortex } \\
\text { (right lateralized) }\end{array}$ & Fox et al., 2006 \\
\hline Salience network & the anterior insula, the dorsal anterior cingulate cortex & Sridharan et al., 2008 22$)$ \\
\hline Executive control network & $\begin{array}{l}\text { the dorsolateral prefrontal cortex, the inferior parietal } \\
\text { cortex, the intraparietal sulcus }\end{array}$ & $\begin{array}{l}\text { Seeley et al., } 2007,^{20)} \\
\text { Koechlin and Summerfield } \\
2007^{21)}\end{array}$ \\
\hline Primary visual network & $\begin{array}{l}\text { the primary visual cortex, the inferior precuneus cortex, } \\
\text { the lateral geniculate nucleus }\end{array}$ & $\begin{array}{l}\text { Beckmann et al., 2005, } \\
\text { De Luca et al., 2006 } \\
\text { 16) }\end{array}$ \\
\hline Higher visual network & $\begin{array}{l}\text { the occipital pole, lateral occipito-temporal junction, } \\
\text { superior parietal lobule }\end{array}$ & $\begin{array}{l}\text { Beckmann et al., 2005, } \\
\text { De Luca et al., 2006 } \\
\end{array}$ \\
\hline Auditory network & $\begin{array}{l}\text { Heschl's gyrus, the lateral superior temporal gyrus, the } \\
\text { posterior insular cortex, anterior cingulate gyrus, the } \\
\text { anterior supramarginal gyrus }\end{array}$ & Smith et al., 2009 ${ }^{17)}$ \\
\hline Sensorimotor network & the precentral gyrus, the posterior central gyrus, the SMA & Biswal et al., $1995^{4)}$ \\
\hline Language network & $\begin{array}{l}\text { the posterior superior temporal gyrus, the marginal gyrus, } \\
\text { the inferior frontal gyrus, the middle temporal gyrus, the } \\
\text { caudate nuclei, the putamen }\end{array}$ & Tomasi and Volkow 2012 ${ }^{18)}$ \\
\hline
\end{tabular}

However, there is no clear reason why low frequency fluctuation is found in such fundamental neural networks. Functional connectivity is characterized predominantly by frequencies $(>0.1 \mathrm{~Hz})$ slower than those in cardiac and respiratory cycles. ${ }^{23)}$ While such low fluctuations can be recorded by digital EEG, it is usually difficult to distinguish them from artifacts, and they tend to be filtered out by the conventional settings for low-cut filters of $0.1 \mathrm{~Hz}$. On the other hand, magnetoencephalography (MEG) recently demonstrated some correlations between low frequency fluctuations and electrophysiological activities. Brookes et al. extracted relatively high frequency activity, such as alpha, beta, and gamma, from MEG datasets, and analyzed it by beam-former spatial filtering and ICA. They successfully proved that the RSNs visualized in MEG data demonstrated significant similarities to those measured by fMRI. In other words, they confirmed that hemodynamic networks analyzed by fMRI truly substantiated neural activities observed with electrophysiological recording. ${ }^{24)}$ Furthermore, recent studies suggest that "cross-frequency coupling" may serve as a mechanism to transfer information from large-scale networks (low frequency fluctuations) to fast, local cortical processing (fast waves) across multiple spatiotemporal scales. ${ }^{25,26)}$ However, further study is required.

\section{Clinical Application in Gliomas}

\section{Alteration of RSNs correlated to cognitive func- tions in gliomas}

Some patients with gliomas or other intracranial tumors show very little or no disturbance in neurological function, even if the tumor is large. Approximately $30 \%$ of patients lack apparent neurological deficits, such as speech disturbance (aphasia) or motor weakness, ${ }^{27}$ ) while the advancement and widespread use of non-invasive imaging technologies (e.g., MRI) has improved the diagnosis of asymptomatic or minimally symptomatic patients, increasing this percentage. However, detailed neuropsychometric tests in these cases often reveal some deficits in cognitive function, including disturbances in comprehension, performance, executive control, working memory, and attention. Such changes in cognition are often difficult to explain when considering only the damage caused by the tumor in focal regions of the brain, but could in fact be network-related. Thus, RSNs were analyzed to evaluate the cortico-subcortical connections in patients with glioma.

Unfortunately, network studies of brain tumor cases are very rare, and only a few papers have been published on this topic. In one study, Harris 


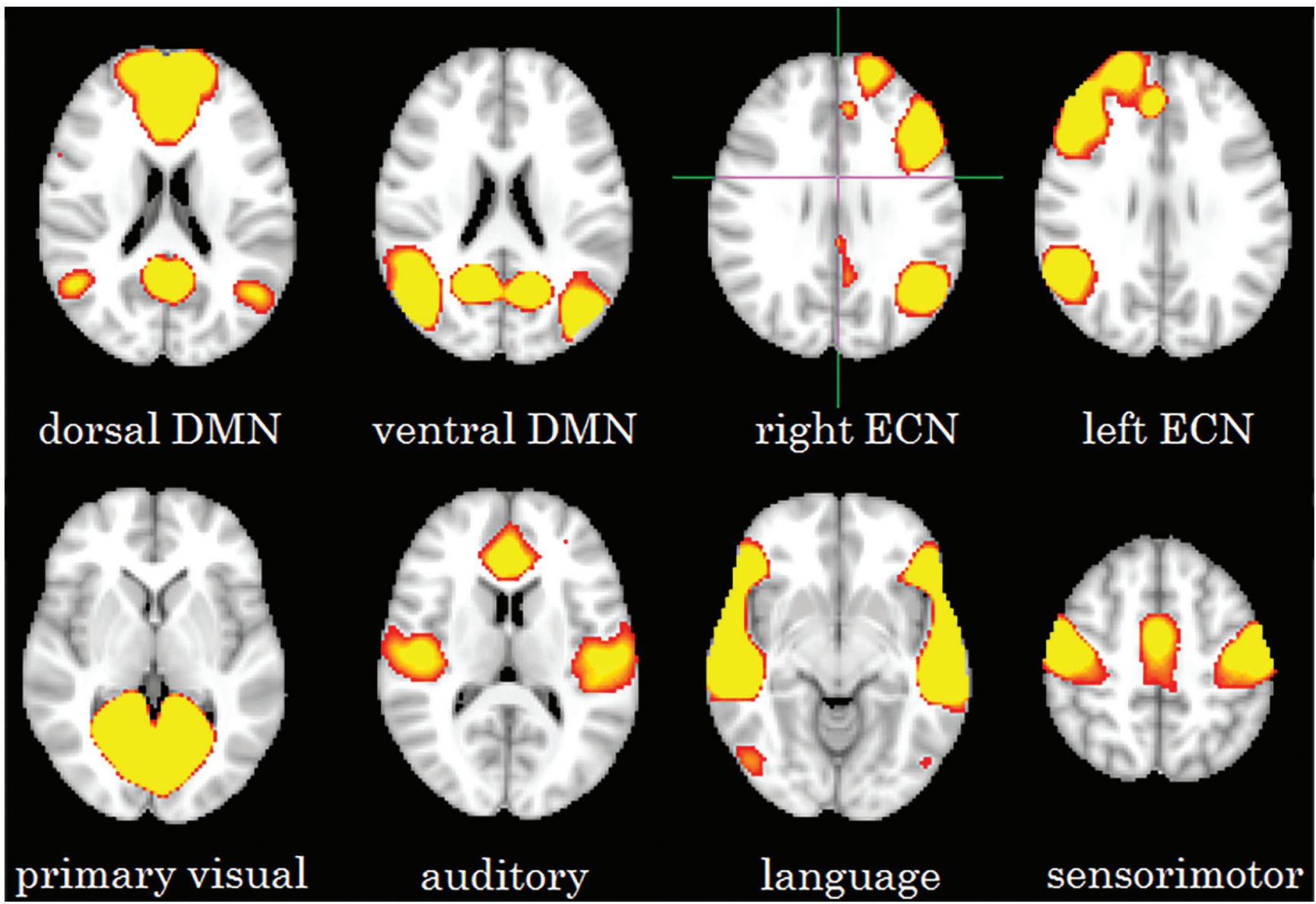

Fig. 1 Representative resting state networks identified by independent component analysis (ICA) with resting state functional magnetic resonance imaging. Resting state functional images were obtained in 125 healthy controls (range of age: 20-59) using a 3.0 Tesla scanner at Nagoya University's Brain and Mind Research Center. After preprocessing, group ICA was performed using the MELODIC software from the FSL package (http://fsl.fmrib. ox.ac.uk/fsl/fslwiki/). We extracted 30 independent components, and identified representative networks. DMN: default mode network, ECN: executive control network.

et al. examined the DMN with pseudo-resting state fMRI (i.e., using datasets derived from task-based fMRI), and showed that DMN integrity decreased in glioma cases. ${ }^{28)}$ The significant predictors of the changes in DMN integrity were WHO grade (highgrade cases showed worsened integrity) and the location of lesions (a lesion in the left parietal lobule was significantly worse than other areas). Esposito et al. studied the DMN with glioma cases using the deactivation profiles of language task datasets, ${ }^{29)}$ and noted increased DMN connectivity in hippocampal lesions, but decreased connectivity in the VMPFC.

Maesawa et al. examined RSNs by ICA with resting state datasets in fMRI in patients with glioma who had little or no neurological functional deficits. These were compared to the scores of representative neuropsychometric examinations for cognition. ${ }^{30)}$ The DMN showed a decrease in functional connectivity in the right angular gyrus/inferior parietal lobe, and the left ECN showed decreased connectivity in the dorsolateral prefrontal cortex; however, the right executive control network showed an increase in connectivity in the right parietal lobe. Moreover, changes in connectivity in the right ECN correlated with spatial memory, while those in the left ECN correlated with attention. Connectivity changes noted in the ventral DMN correlated with attention, working memory, and verbal intelligence quotient measures. These data indicate that patients with gliomas may have some cognitive changes related to alterations across wide-area brain networks, even in the absence of major symptoms. This could potentially explain some of the behavioral changes often seen in glioma cases, such as slowness of reaction, somnolence, loss of attention, and working memory problems. Thus, evaluation of RSNs is potentially 
helpful in advancing our hodological understanding of brain function in glioma cases.

\section{Presurgical localization of eloquent cortex}

Resting state fMRI requires no explicit task for the participant to perform during the scan, and is therefore highly suitable for preoperative evaluation in a patient population who might be less responsive or unable to respond in parallel to healthy controls because of particular task designs. Several approaches have been reported for the application of resting state fMRI for preoperative planning. Zhang et al. reported that the localization of the sensorimotor cortex was enabled using a seed-based approach with resting state fMRI. In this approach, an ROI was set in the sensorimotor region in the unaffected hemisphere, and functional connectivity to the diseased hemisphere was calculated in patients with a glioma-distorted hemisphere. ${ }^{31)}$ Kokkonen et al. demonstrated that the sensorimotor areas localized by ICA through datasets of resting state fMRI were highly consistent with those using datasets of task-based fMRI. ${ }^{32)}$ Identification of the language area in the glioma-distorted brain is more challenging, as its location is known to be more variable. ${ }^{33)}$ Recently, Mitchell et al. evaluated the usefulness of a data-driven approach with resting state fMRI for preoperative functional mapping, including language-dominant areas. They identified the RSNs by an artificial neural network algorithm, compared these to the results of intraoperative mapping with electrical stimulation, and concluded that this approach was effective in non-invasive preoperative localization. ${ }^{34)}$ We have studies ongoing that will evaluate the possibility of the identification of language-dominant areas by ICA (Fig. 2); however, further evaluation has been required, including a comparison with intraoperative mapping by electrical stimulation.

3. Glioma grading measured by resting state fMRI Another application of resting state fMRI is in the grading of gliomas. Malignant gliomas demonstrate a high degree of tumor cell infiltration and proliferation compared to low-grade gliomas. These characteristics could be potentially quantified by advanced analysis of non-invasive resting state fMRIs. In one recent investigation, Harris et al. evaluated the DMN in 68 glioma patients, and concluded that higher tumor grading correlated with the largest reductions in DMN functional connectivity. ${ }^{28)}$ On the other hand, Esposito et al. obtained contrary results, ${ }^{29)}$ noting that the decrease in connectivity of the DMN was often observed in low-grade gliomas rather than in high-grade gliomas. They hypothesized that the lack of aggressive growth of low-grade gliomas may permit a more marked plastic reorganization of neural networks, resulting in greater alteration of the DMN. Although these opposing results could be explained by the differences in methodology and types of datasets used, further evaluations are necessary to better elucidate the relationship between the tumor grading and the functional connectivity to the DMN. Finally, malignant gliomas are associated with infiltration into the surrounding brain parenchyma, resulting in damage to regions of the brain that may be involved in particular neurological functions. Though no data regarding this concept were identified, it is conceivable that functional connectivity with resting state fMRI may provide additional insight into the degree of the tumor infiltration without requiring biopsy or other invasive techniques.

There is another approach to estimate the tumor grade using resting state fMRI, very recently reported by $\mathrm{Wu}$ et al. ${ }^{35}$ ) This approach focused on several parameters described by resting state fMRI, which may reflect anatomical morphology in tumor regions. Such parameters include the signal intensity difference ratio, signal intensity correlation (SIC), factional amplitude of low frequency fluctuation (fALFF), and regional homogeneity (ReHo). When a support vector machine was employed to distinguish high- and lowgrade gliomas, it was found that high-grade gliomas demonstrated more complex anatomical morphology as defined in particular by SIC, fALFF, and ReHo. The accuracy, sensitivity, and specificity of classification with support vector machine were greater than $80 \%$. For tumor grading, tumor heterogeneity must be considered, since the tumors consist of various tissues, including necrotic/cystic areas, hemorrhage, or infiltrative edema. Such varying tissue types would have distinguishable parameters using resting state fMRI, and would need to be combined for tumor grading. Furthermore, the association of tumor grading in this manner and disease prognosis should be evaluated.

\section{Clinical Applications in Epilepsy}

\section{Localization of the epileptic focus or network} Epilepsy is estimated to have a prevalence of $0.5-1 \%$ in the entire population. In this group, $30 \%$ of the patients with epilepsy were medically uncontrollable, and their daily life was impaired to varying degrees. ${ }^{36)}$ For medically refractory epilepsy, surgical resection is a necessary approach, and precise identification of the epileptic focus, as well as nearby functional regions, is critical in achieving favorable outcomes. However, there is a need for less invasive and more reliable diagnostic tools for identification of epileptic foci. Recent studies suggest resting state fMRI with 


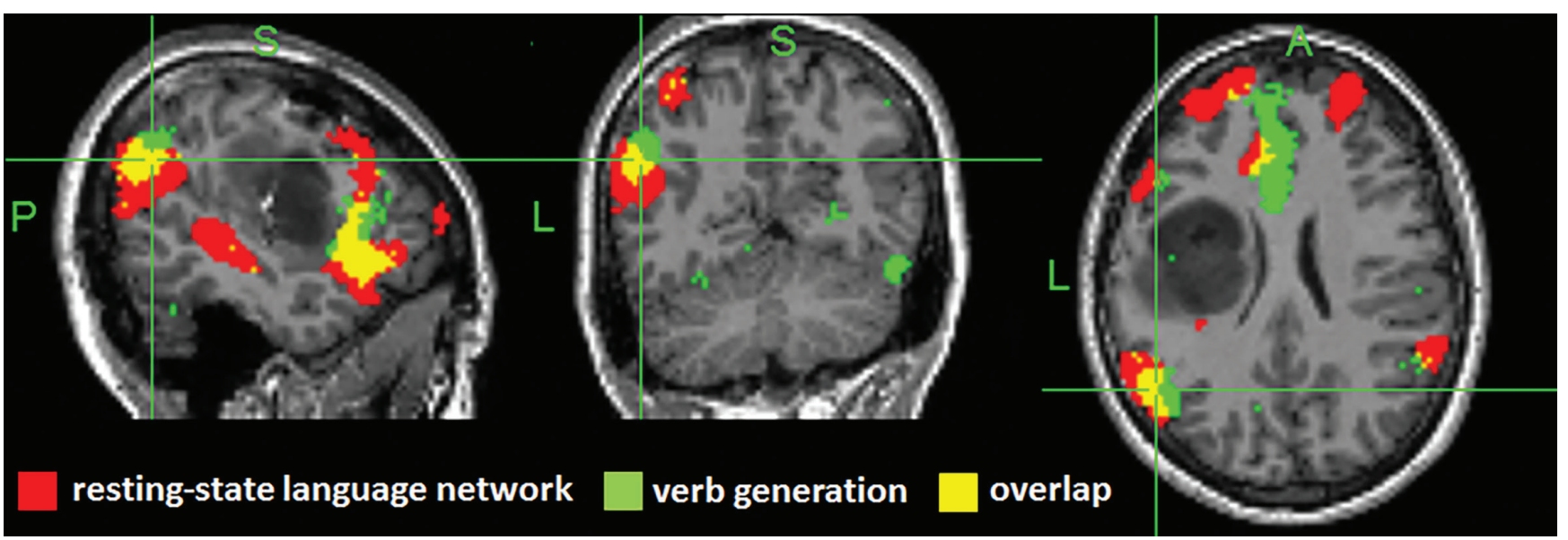

Fig. 2 The comparison of the language networks in resting state fMRI and task fMRI in a patient with glioma. The patient was a 35-year-old female who had anaplastic astrocytoma in the left insula. Individual independent component analysis was performed with the datasets of resting state fMRI (red) and fMRI with a verb generation task (green). Although the affected hemisphere was distorted, the language networks with both methods appeared in the appropriate language-related regions, including Broca's and Wernicke's areas, with overlapping regions in both areas (yellow) (unpublished data). fMRI: functional magnetic resonance imaging.

various computational analyses could represent a novel diagnostic tool for such a purpose.

Several studies have been performed to utilize this technique for focus detection in a series of patients with epilepsy. For example, Bettus et al. utilized functional connectivity analysis by resting state fMRI in patients with mesial temporal lobe epilepsy (TLE) ${ }^{37)}$ They examined functional connectivity between 10 potentially epileptogenic regions, including the hippocampus, the amygdala, and the entorhinal cortex, and found that functional connectivity was decreased in the hemisphere with the epileptic focus, whereas it was increased in the contralateral side. This may be a compensatory effect in the contralateral side, mitigating damage from repeated seizures in the damaged side. This rightleft imbalance could be useful in identifying the side of the epileptic focus in mesial TLE patients, although in the above study epileptic focus was not directly examined. Stufflebeam et al. analyzed functional connectivity in a series of patients with medically refractory epilepsy in whom an identifiable lesion had not been imaged. ${ }^{38}$ They found an increase in functional connectivity in and around the locations with the suspected epileptic focus, compared to that in 300 healthy controls. They hypothesized that functional connectivity increased closer to the epileptogenic focus, in contradiction of the data and hypothesis put forth in the above study by Bettus et al. This discrepancy might be explained by differences in the subject groups, in the disease pathology, or in the disease stage, and as of this writing remains unresolved.
Zhang et al. reported a further comprehensive evaluation of neural networks, in this case applying the social network theory in patients with MTLE. ${ }^{39)}$ They revealed that the pattern classifier by graph methods could reveal abnormal network properties in epilepsy patients at the group level. More recently, Dansereau et al. proposed a novel analysis method, "detection of abnormal networks in individuals (DANI)," to identify modularity changes at the individual level. ${ }^{40)}$ They evaluated the stability of each RSN, and detected abnormal individual changes in network organization in patients with focal epilepsy when compared to healthy controls. They concluded that at least one abnormally lateralized network closely related to the epileptic focus was successfully detected.

Thus, although the methodology varied among these studies, some progress has been made by focusing on the changes in neural networks in epilepsy, assuming that such approaches may better elucidate the relationship between the epileptic focus and pathways of propagation in epilepsy in the near future. Important issues remain, though, including how to discriminate between decreases in normal networks and those in newly formed networks created by repeated seizures; as well as how to perform the above types of network analyses during periods with or without spikes.

\section{Epilepsy-associated networks studied with EEG-fMRI \\ Simultaneous recording of EEG and fMRI (EEG-fMRI) is a technique that can reveal the regions of the brain}


showing changes in blood flow and metabolism in response to interictal epileptic discharges (IEDs) identified in EEG. It is based on the hypothesis that fMRI signals in the regions where spikes originate presumably reflect the intense neuronal activity of the seizure discharge, similarly to SPECT studies performed at the time of epileptic seizures to determine the regions of increased blood flow. ${ }^{41,42)}$

The pioneering study of Ives et al. demonstrated that it is possible to record the EEG in such a hostile environment. ${ }^{43)}$ The basic method of EEG-fMRI recording begins with using nonferrous electrodes and leads, and perhaps current-limiting resistors, for EEG. ${ }^{44)}$ In addition, current loops involving the patient should be avoided. ${ }^{45}$ During fMRI scanning, the rapidly changing magnetic fields induce currents in the electrodes and leads, resulting in EEG artifacts ("gradient artifacts"). The gradient artifacts are removed by methods originally proposed by Allen et al., which consist of artifact estimation, subtraction from each frame, and adaptive noise cancellation. ${ }^{46)}$ Recently, several variations on the subtraction methods have been proposed. ${ }^{47-49)}$ Another problematic artifact is a "ballistocardiogram artifact," which may be caused by small movements of the head or electrodes related to the fast movement of blood in the arteries following each pulsation. ${ }^{43}$ It can be removed by averaging and subtraction, ${ }^{50)}$ adaptive filtering, ${ }^{51)}$ wavelet filtering, ${ }^{52)}$ or ICA. ${ }^{53)}$ After artifact removal, event-related analysis is used to identify epileptic events, by correlating the timing of the spikes (the events) with the hemodynamic response function (HRF). Although a standard HRF, the measured response to a brief stimulus such as an auditory tone, ${ }^{54)}$ has been widely used, several different approaches were evaluated using variable shape and timing of the modeled HRF. ${ }^{55,56)}$ Here, we provide an example of a case of focal epilepsy with a lesion in the anterior cingulate gyrus to demonstrate evaluation by EEG-fMRI (Fig. 3).

In clinical epilepsy studies, a Montreal Neurological Institute group led by Gotman examined a series of TLE patients, and reported that epilepsyrelated BOLD responses occurred in $83 \%$ of patients, predominantly in the temporal lobe. ${ }^{57)}$ They also evaluated a series of patients with gray matter heterotopia, and reported that some signal clusters are seen in or very close to the lesions, although not consistently. ${ }^{58)}$ Idiopathic generalized epilepsy (IGE) was also studied, ${ }^{59)}$ and thalamic activation was identified in concert with widespread, bilateral, and symmetrical deactivation of the cerebral cortex. Gotman et al. reported that bilateral activation was found in the thalamus, mesial frontal region, insula, and middle cerebellum, whereas deactivations were found bilaterally in the anterior frontal and parietal regions and in the posterior cingulate gyri. Note that the latter regions are components of the "default mode network." ${ }^{60)}$

The sensitivity of EEG-fMRI is limited by the detection power of IEDs with scalp EEG, and the suitability of the modeling of the fMRI signal. ${ }^{61)}$ In order to improve the sensitivity, Grouiller et al. have suggested the initial construction of topographic maps from IEDs recorded during long-term video-EEG monitoring. ${ }^{62)}$ Although few in number, initial reports in which intracranial EEG-fMRI was performed after implantation of intracranial electrodes demonstrated $100 \%$ sensitivity in identifying IED-related BOLD changes. ${ }^{63-65)}$

The relationship between the locations of IEDrelated BOLD changes and surgical outcome has been examined, showing that when surgical resection includes regions with IED-related BOLD changes, there is a high positive predictive value for a good postoperative outcome, which declines with widespread or discordant changes. ${ }^{6-68)}$

\section{Alteration in RSNs in TLE}

Significant advances in the analysis of RSNs represent a chance to investigate the still unclear pathophysiology of the disabling cognitive and psychiatric manifestations of TLE. In 2010, Zhang et al. demonstrated that the overall area of activation of the DMN was reduced in patients with TLE, especially in the medial prefrontal structures. ${ }^{69)}$ One possible mechanism for this decrease in connectivity is the accumulation of structural damage due to repeated seizures. This hypothesis was supported by diffusion tensor imaging (DTI), which identified relevant structural damage in white matter bundles connecting the distant regions of the DMN. ${ }^{70)}$ Other functional connectivity studies have demonstrated positive correlations with disease years ${ }^{71)}$ or severity of hippocampal sclerosis. ${ }^{72)}$ Since activities such as abstract thinking, cognition, and memory retrieval are regulated by the $\mathrm{DMN}$, alterations in those processes could be observed in patients with mesial TLE, as well as Alzheimer disease, autism, and attention deficit/hyperactivity disorder. ${ }^{73,74)}$

Two main attention networks, the ventral and dorsal attention networks, are reportedly altered in TLE patients. The dorsal attention network mediates topdown attention, or attention directed towards specific subjects, ${ }^{75)}$ had significant decrements in functional connectivity in patients with mesial TLE. ${ }^{76)}$ The ventral attention network mediates bottom-up attention or attentiveness triggered by sudden, unexpected stimuli; it too was reported to be impaired in patients with TLE in a study combining P300 wave analysis with high-density EEG recording. ${ }^{77)}$ Furthermore, decreases in 

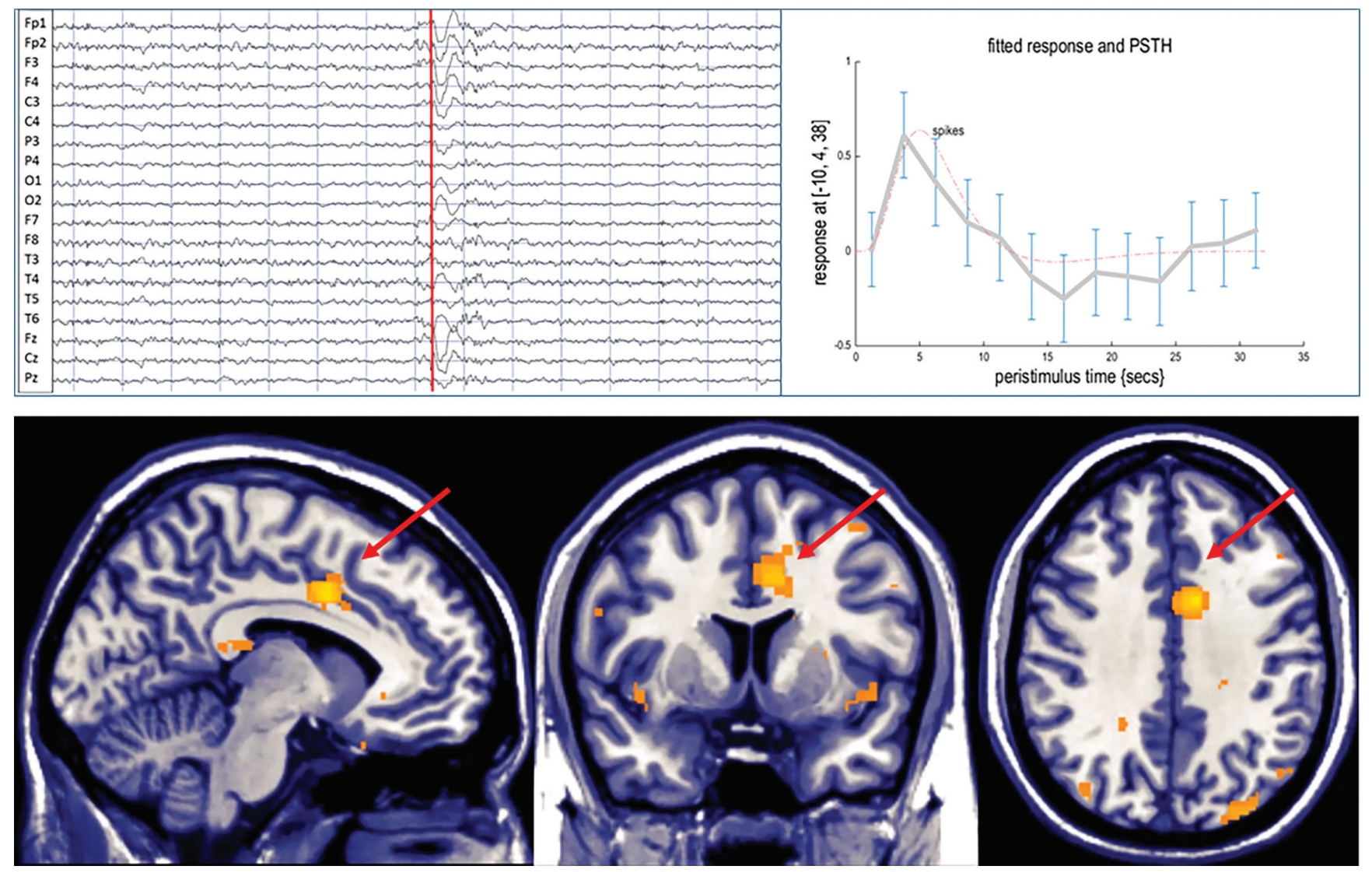

Fig. 3 An interictal epileptic discharge (IED)-related cluster in the EEG-fMRI of a patient with focal epilepsy. The patient was a 19-year-old female who had medically refractory epilepsy with a lesion in the left anterior cingulate gyrus. The IEDs were observed maximally in the CZ and FZ electrodes during the recording of the EEG simultaneously with MRI scanning (left upper panel); the event-related analysis was performed at the times of occurrence of 25 observed IEDs (right upper panel). An IED-related cluster was successfully identified in and near the lesion (lower panel). EEG-fMRI: electroencephalography functional magnetic resonance imaging, PSTH: Peri-Stimulus Time Histogram.

functional connectivity were reported in the executive control network, ${ }^{78)}$ which is thought to be involved in schizophrenia and typical psychosis. ${ }^{79)}$ Thus, it could be involved in the genesis of the so-called psychosis of epilepsy. ${ }^{78)}$ Together, the reports described here indicate that the dysfunction of RSNs could have an important role in the occurrence of cognitive and psychiatric complications of TLE.

\section{Future Directions}

Two topics are introduced here that reflect the potential of application of resting state fMRI study. The first is its capacity to identify individualized functional localization in pre-surgical settings. To date, a precise functional mapping still relies heavily on invasive measures.

Although network analysis with resting state fMRI has mainly focused on evaluation of large-scale networks so far, big data analysis of healthy controls has brought about quantification and visualization of the individual variation for functional localization.

Very recently, Wang et al. reported the promising method of non-invasive functional localization using an iterative cortical parcellation approach using resting state fMRI ${ }^{80)}$ A functional cortical atlas was developed using datasets from 1,000 healthy controls, ${ }^{81)}$ and a map of inter-individual variability was employed to guide the iterative search for functional networks in individual subjects. Functional networks mapped by this approach demonstrated high reproducibility and high detection power for variability across the patients. Furthermore, they found that brain lateralization is reflected in the network parcellation, especially that of language-dominant areas. Although one limitation of this approach is its application in patients with disturbed brain anatomy, establishment of this approach could lead to the successful creation of a non-invasive functional map of the whole brain for individual patients. The functional map made by this 
approach is advantageous with regard to its ability to be less invasive, but also the benefit of providing additional information on functional connectivity to other regions. The comprehensive interpretation of functional connectivity among regions contributes to building up a more sophisticated surgical plan for gliomas or for epilepsy surgery, to a level wellsuited to a hodological approach.

The second concept is the combination of other modalities with resting state fMRI. The network information obtained in this manner has been combined with EEG already, but could conceivably be combined with the simultaneous recording of other modalities, including high resolution structural MRI, DTI, PET, or MEG. These combined methods could elucidate many different aspects of the brain at once, including morphology, electrophysiology, blood circulation, and metabolism. This could lead to a more comprehensive understanding of brain function without the need for animal modeling or invasive clinical studies.

\section{Conclusion}

Resting state fMRI has the potential to provide new insights on the functional aspects of the human brain. The analysis of RSNs using this technique has made it possible to evaluate brain function noninvasively in patients scheduled for surgery for gliomas and epilepsy. It has been applied to the identification of areas with dominant functions such as language or sensorimotor functions, although it can be limited in this sense by the distorted hemisphere. The DMN, a representative RSN, has been evaluated with resting state fMRI, and found to have impairments in both of the above patient groups correlated with cognitive function or psychosis. Detection of epileptic foci using resting state fMRI is still challenging; however, when combined with the simultaneous recording of EEG, the dual approach demonstrated promising results. For best results, a hodological approach should be taken in establishing the parameters for surgery for gliomas and epilepsy, and through improvements in network analysis and resting state fMRI, the overall approach and neurosurgical environment should continue to advance as well.

\section{Acknowledgments}

This study was supported by a Grant-in-Aid for Scientific Research (KAKENHI, no. 26462202).

\section{Conflicts of Interest Disclosure}

The authors declare that there is no conflict of interest (COI) regarding this article according to the criteria of The Japan Neurosurgical Society. They completed the self-reported registration of their COI status to the society.

\section{References}

1) Van Essen DC, Ugurbil K, Auerbach E, Barch D, Behrens TE, Bucholz R, Chang A, Chen L, Corbetta M, Curtiss SW, Della Penna S, Feinberg D, Glasser MF, Harel N, Heath AC, Larson-Prior L, Marcus D, Michalareas G, Moeller S, Oostenveld R, Petersen SE, Prior F, Schlaggar BL, Smith SM, Snyder AZ, $\mathrm{Xu}$ J, Yacoub E; WU-Minn HCP Consortium: The Human Connectome Project: a data acquisition perspective. Neuroimage 62: 2222-2231, 2012

2) Catani M: From hodology to function. Brain 130: 602-605, 2007

3) Bennett CM, Miller MB: How reliable are the results from functional magnetic resonance imaging? Ann N Y Acad Sci 1191: 133-155, 2010

4) Biswal B, Yetkin FZ, Haughton VM, Hyde JS: Functional connectivity in the motor cortex of resting human brain using echo-planar MRI. Magn Reson Med 34: 537-541, 1995

5) Raichle ME, MacLeod AM, Snyder AZ, Powers WJ, Gusnard DA, Shulman GL: A default mode of brain function. Proc Natl Acad Sci USA 98: 676-682, 2001

6) Greicius MD, Krasnow B, Reiss AL, Menon V: Functional connectivity in the resting brain: a network analysis of the default mode hypothesis. Proc Natl Acad Sci U S A 100: 253-258, 2003

7) Shehzad Z, Kelly AM, Reiss PT, Gee DG, Gotimer K, Uddin LQ, Lee SH, Margulies DS, Roy AK, Biswal BB, Petkova E, Castellanos FX, Milham MP: The resting brain: unconstrained yet reliable. Cereb Cortex 19: 2209-2229, 2009

8) McKiernan KA, D’Angelo BR, Kaufman JN, Binder JR: Interrupting the "stream of consciousness": an fMRI investigation. Neuroimage 29: 1185-1191, 2006

9) Goldberg II, Harel M, Malach R: When the brain loses its self: prefrontal inactivation during sensorimotor processing. Neuron 50: 329-339, 2006

10) Spreng RN, Mar RA, Kim AS: The common neural basis of autobiographical memory, prospection, navigation, theory of mind, and the default mode: a quantitative meta-analysis. J Cogn Neurosci 21: 489-510, 2009

11) Supekar K, Menon V, Rubin D, Musen M, Greicius MD: Network analysis of intrinsic functional brain connectivity in Alzheimer's disease. PLoS Comput Biol 4: e1000100, 2008

12) Zhu DC, Majumdar S, Korolev IO, Berger KL, Bozoki AC: Alzheimer's disease and amnestic mild cognitive impairment weaken connections within the default-mode network: a multi-modal imaging study. J Alzheimers Dis 34: 969-984, 2013

13) Waltz JA, Kasanova Z, Ross TJ, Salmeron BJ, McMahon RP, Gold JM, Stein EA: The roles of reward, default, and executive control networks 
in set-shifting impairments in schizophrenia. PLoS ONE 8: e57257, 2013

14) Filippi M, Agosta F, Scola E, Canu E, Magnani G, Marcone A, Valsasina P, Caso F, Copetti M, Comi G, Cappa SF, Falini A: Functional network connectivity in the behavioral variant of frontotemporal dementia. Cortex 49: 2389-2401, 2013

15) Beckmann CF, DeLuca M, Devlin JT, Smith SM: Investigations into resting-state connectivity using independent component analysis. Philos Trans $R$ Soc Lond B Biol Sci 360: 1001-1013, 2005

16) De Luca M, Beckmann CF, De Stefano N, Matthews PM, Smith SM: fMRI resting state networks define distinct modes of long-distance interactions in the human brain. Neuroimage 29: 1359-1367, 2006

17) Smith SM, Fox PT, Miller KL, Glahn DC, Fox PM, Mackay CE, Filippini N, Watkins KE, Toro R, Laird AR, Beckmann CF: Correspondence of the brain's functional architecture during activation and rest. Proc Natl Acad Sci USA 106: 13040-13045, 2009

18) Tomasi D, Volkow ND: Resting functional connectivity of language networks: characterization and reproducibility. Mol Psychiatry 17: 841-854, 2012

19) Fox MD, Corbetta M, Snyder AZ, Vincent JL, Raichle ME: Spontaneous neuronal activity distinguishes human dorsal and ventral attention systems. Proc Natl Acad Sci USA 103: 10046-10051, 2006

20) Seeley WW, Menon V, Schatzberg AF, Keller J, Glover GH, Kenna H, Reiss AL, Greicius MD: Dissociable intrinsic connectivity networks for salience processing and executive control. J Neurosci 27: 2349-2356, 2007

21) Koechlin E, Summerfield C: An information theoretical approach to prefrontal executive function. Trends Cogn Sci (Regul Ed) 11: 229-235, 2007

22) Sridharan D, Levitin DJ, Menon V: A critical role for the right fronto-insular cortex in switching between central-executive and default-mode networks. Proc Natl Acad Sci USA 105: 12569-12574, 2008

23) Cordes D, Haughton VM, Arfanakis K, Carew JD, Turski PA, Moritz CH, Quigley MA, Meyerand ME: Frequencies contributing to functional connectivity in the cerebral cortex in "resting-state" data. AJNR Am J Neuroradiol 22: 1326-1333, 2001

24) Brookes MJ, Woolrich M, Luckhoo H, Price D, Hale JR, Stephenson MC, Barnes GR, Smith SM, Morris PG: Investigating the electrophysiological basis of resting state networks using magnetoencephalography. Proc Natl Acad Sci USA 108: 16783-16788, 2011

25) Foster BL, Parvizi J: Resting oscillations and crossfrequency coupling in the human posteromedial cortex. Neuroimage 60: 384-391, 2012

26) Canolty RT, Knight RT: The functional role of crossfrequency coupling. Trends Cogn Sci (Regul Ed) 14: 506-515, 2010

27) Mahaley MS Jr, Mettlin C, Natarajan N, Laws ER Jr, Peace BB: National survey of patterns of care for brain-tumor patients. J Neurosurg 71: 826-836, 1989

28) Harris RJ, Bookheimer SY, Cloughesy TF, Kim HJ, Pope WB, Lai A, Nghiemphu PL, Liau LM,
Ellingson BM: Altered functional connectivity of the default mode network in diffuse gliomas measured with pseudo-resting state fMRI. J Neurooncol 116: 373-379, 2014

29) Esposito R, Mattei PA, Briganti C, Romani GL, Tartaro A, Caulo M: Modifications of default-mode network connectivity in patients with cerebral glioma. PLoS ONE 7: e40231, 2012

30) Maesawa S, Bagarinao E, Fujii M, Futamura M, Motomura K, Watanabe H, Mori D, Sobue G, Wakabayashi T: Evaluation of resting state networks in patients with gliomas: connectivity changes in the unaffected side and its relation to cognitive function. PLoS ONE 10: e0118072, 2015

31) Zhang D, Johnston JM, Fox MD, Leuthardt EC, Grubb RL, Chicoine MR, Smyth MD, Snyder AZ, Raichle ME, Shimony JS: Preoperative sensorimotor mapping in brain tumor patients using spontaneous fluctuations in neuronal activity imaged with functional magnetic resonance imaging: initial experience. Neurosurgery 65: 226-236, 2009

32) Kokkonen SM, Nikkinen J, Remes J, Kantola J, Starck T, Haapea M, Tuominen J, Tervonen O, Kiviniemi V: Preoperative localization of the sensorimotor area using independent component analysis of restingstate fMRI. Magn Reson Imaging 27: 733-740, 2009

33) Sanai N, Mirzadeh Z, Berger MS: Functional outcome after language mapping for glioma resection. $N$ Engl J Med 358: 18-27, 2008

34) Mitchell TJ, Hacker CD, Breshears JD, Szrama NP, Sharma M, Bundy DT, Pahwa M, Corbetta M, Snyder AZ, Shimony JS, Leuthardt EC: A novel data-driven approach to preoperative mapping of functional cortex using resting-state functional magnetic resonance imaging. Neurosurgery 73: 969-982; discussion 982-983, 2013

35) Wu J, Qian Z, Tao L, Yin J, Ding S, Zhang Y, Yu $\mathrm{Z}$ : Resting state fMRI feature-based cerebral glioma grading by support vector machine. Int J Comput Assist Radiol Surg 10: 1167-1174, 2015

36) Litt B, Echauz J: Prediction of epileptic seizures. Lancet Neurol 1: 22-30, 2002

37) Bettus G, Guedj E, Joyeux F, Confort-Gouny S, Soulier E, Laguitton V, Cozzone PJ, Chauvel P, Ranjeva JP, Bartolomei F, Guye M: Decreased basal fMRI functional connectivity in epileptogenic networks and contralateral compensatory mechanisms. Hum Brain Mapp 30: 1580-1591, 2009

38) Stufflebeam SM, Liu H, Sepulcre J, Tanaka N, Buckner RL, Madsen JR: Localization of focal epileptic discharges using functional connectivity magnetic resonance imaging. J Neurosurg 114: 1693-1697, 2011

39) Zhang X, Tokoglu F, Negishi M, Arora J, Winstanley S, Spencer DD, Constable RT: Social network theory applied to resting-state fMRI connectivity data in the identification of epilepsy networks with iterative feature selection. J Neurosci Methods 199: 129-139, 2011

40) Dansereau CL, Bellec P, Lee K, Pittau F, Gotman J, Grova C: Detection of abnormal resting-state networks in individual patients suffering from focal 
epilepsy: an initial step toward individual connectivity assessment. Front Neurosci 8: 419, 2014

41) Newton MR, Austin MC, Chan JG, McKay WJ, Rowe CC, Berkovic SF: Ictal SPECT using technetium99m-HMPAO: methods for rapid preparation and optimal deployment of tracer during spontaneous seizures. J Nucl Med 34: 666-670, 1993

42) Berkovic SF: SPECT: neurobiology of periictal blood flow alterations. Adv Neurol 83: 33-39, 2000

43) Ives JR, Warach S, Schmitt F, Edelman RR, Schomer DL: Monitoring the patient's EEG during echo planar MRI. Electroencephalogr Clin Neurophysiol 87: 417-420, 1993

44) Lemieux L, Allen PJ, Franconi F, Symms MR, Fish DR: Recording of EEG during fMRI experiments: patient safety. Magn Reson Med 38: 943-952, 1997

45) Lazeyras F, Zimine I, Blanke O, Perrig SH, Seeck M: Functional MRI with simultaneous EEG recording: feasibility and application to motor and visual activation. J Magn Reson Imaging 13: 943-948, 2001

46) Allen PJ, Josephs O, Turner R: A method for removing imaging artifact from continuous EEG recorded during functional MRI. Neuroimage 12: 230-239, 2000

47) Goldman RI, Stern JM, Engel J Jr, Cohen MS: Acquiring simultaneous EEG and functional MRI. Clin Neurophysiol 111: 1974-1980, 2000

48) Garreffa G, Carnì M, Gualniera G, Ricci GB, Bozzao L, De Carli D, Morasso P, Pantano P, Colonnese C, Roma V, Maraviglia B: Real-time MR artifacts filtering during continuous EEG/fMRI acquisition. Magn Reson Imaging 21: 1175-1189, 2003

49) Negishi M, Abildgaard M, Nixon T, Constable RT: Removal of time-varying gradient artifacts from EEG data acquired during continuous fMRI. Clin Neurophysiol 115: 2181-2192, 2004

50) Allen PJ, Polizzi G, Krakow K, Fish DR, Lemieux L: Identification of EEG events in the MR scanner: the problem of pulse artifact and a method for its subtraction. Neuroimage 8: 229-239, 1998

51) Bonmassar G, Purdon PL, Jääskeläinen IP, Chiappa K, Solo V, Brown EN, Belliveau JW: Motion and ballistocardiogram artifact removal for interleaved recording of EEG and EPs during MRI. Neuroimage 16: 1127-1141, 2002

52) Kim KH, Yoon HW, Park HW: Improved ballistocardiac artifact removal from the electroencephalogram recorded in fMRI. J Neurosci Methods 135: 193-203, 2004

53) Bénar C, Aghakhani Y, Wang Y, Izenberg A, Al-Asmi A, Dubeau F, Gotman J: Quality of EEG in simultaneous EEG-fMRI for epilepsy. Clin Neurophysiol 114: 569-580, 2003

54) Glover GH: Deconvolution of impulse response in event-related BOLD fMRI. Neuroimage 9: 416-429, 1999

55) Friston KJ, Fletcher P, Josephs O, Holmes A, Rugg MD, Turner R: Event-related fMRI: characterizing differential responses. Neuroimage 7: 30-40, 1998

56) Bagshaw AP, Aghakhani Y, Bénar CG, Kobayashi E, Hawco C, Dubeau F, Pike GB, Gotman J: EEG-fMRI of focal epileptic spikes: analysis with multiple haemodynamic functions and comparison with gadolinium-enhanced MR angiograms. Hum Brain Mapp 22: 179-192, 2004

57) Kobayashi E, Bagshaw AP, Bénar CG, Aghakhani Y, Andermann F, Dubeau F, Gotman J: Temporal and extratemporal BOLD responses to temporal lobe interictal spikes. Epilepsia 47: 343-354, 2006

58) Kobayashi E, Bagshaw AP, Grova C, Gotman J, Dubeau F: Grey matter heterotopia: what EEG-fMRI can tell us about epileptogenicity of neuronal migration disorders. Brain 129: 366-374, 2006

59) Aghakhani Y, Bagshaw AP, Bénar CG, Hawco C, Andermann F, Dubeau F, Gotman J: fMRI activation during spike and wave discharges in idiopathic generalized epilepsy. Brain 127: 1127-1144, 2004

60) Gotman J, Grova C, Bagshaw A, Kobayashi E, Aghakhani Y, Dubeau F: Generalized epileptic discharges show thalamocortical activation and suspension of the default state of the brain. Proc Natl Acad Sci USA 102: 15236-15240, 2005

61) Salek-Haddadi A, Diehl B, Hamandi K, Merschhemke M, Liston A, Friston K, Duncan JS, Fish DR, Lemieux L: Hemodynamic correlates of epileptiform discharges: an EEG-fMRI study of 63 patients with focal epilepsy. Brain Res 1088: 148-166, 2006

62) Grouiller F, Thornton RC, Groening K, Spinelli L, Duncan JS, Schaller K, Siniatchkin M, Lemieux L, Seeck M, Michel CM, Vulliemoz S: With or without spikes: localization of focal epileptic activity by simultaneous electroencephalography and functional magnetic resonance imaging. Brain 134: 2867-2886, 2011

63) Chaudhary UJ, Carmichael DW, Rodionov R, Thornton RC, Bartlett P, Vulliemoz S, Micallef C, McEvoy AW, Diehl B, Walker MC, Duncan JS, Lemieux L: Mapping preictal and ictal haemodynamic networks using video-electroencephalography and functional imaging. Brain 135: 3645-3663, 2012

64) Vulliemoz S, Carmichael DW, Rosenkranz K, Diehl B, Rodionov R, Walker MC, McEvoy AW, Lemieux L: Simultaneous intracranial EEG and fMRI of interictal epileptic discharges in humans. Neuroimage 54: 182-190, 2011

65) Cunningham CB, Goodyear BG, Badawy R, Zaamout F, Pittman DJ, Beers CA, Federico P: Intracranial EEG-fMRI analysis of focal epileptiform discharges in humans. Epilepsia 53: 1636-1648, 2012

66) Thornton R, Laufs H, Rodionov R, Cannadathu S, Carmichael DW, Vulliemoz S, Salek-Haddadi A, McEvoy AW, Smith SM, Lhatoo S, Elwes RD, Guye M, Walker MC, Lemieux L, Duncan JS: EEG correlated functional MRI and postoperative outcome in focal epilepsy. J Neurol Neurosurg Psychiatr 81: 922-927, 2010

67) Thornton R, Vulliemoz S, Rodionov R, Carmichael DW, Chaudhary UJ, Diehl B, Laufs H, Vollmar C, McEvoy AW, Walker MC, Bartolomei F, Guye M, Chauvel P, Duncan JS, Lemieux L: Epileptic networks in focal cortical dysplasia revealed using 
electroencephalography-functional magnetic resonance imaging. Ann Neurol 70: 822-837, 2011

68) An D, Fahoum F, Hall J, Olivier A, Gotman J, Dubeau F: Electroencephalography/functional magnetic resonance imaging responses help predict surgical outcome in focal epilepsy. Epilepsia 54: 2184-2194, 2013

69) Zhang Z, Lu G, Zhong Y, Tan Q, Chen H, Liao W, Tian L, Li Z, Shi J, Liu Y: fMRI study of mesial temporal lobe epilepsy using amplitude of low-frequency fluctuation analysis. Hum Brain Mapp 31: 1851-1861, 2010

70) Liao W, Zhang Z, Pan Z, Mantini D, Ding J, Duan X, Luo C, Wang Z, Tan Q, Lu G, Chen H: Default mode network abnormalities in mesial temporal lobe epilepsy: a study combining fMRI and DTI. Hum Brain Mapp 32: 883-895, 2011

71) Liao W, Zhang Z, Pan Z, Mantini D, Ding J, Duan X, Luo C, Lu G, Chen H: Altered functional connectivity and small-world in mesial temporal lobe epilepsy. PLoS ONE 5: e8525, 2010

72) Voets NL, Beckmann CF, Cole DM, Hong S, Bernasconi A, Bernasconi N: Structural substrates for resting network disruption in temporal lobe epilepsy. Brain 135: 2350-2357, 2012

73) Laufs H, Hamandi K, Salek-Haddadi A, Kleinschmidt AK, Duncan JS, Lemieux L: Temporal lobe interictal epileptic discharges affect cerebral activity in "default mode" brain regions. Hum Brain Mapp 28: 1023-1032, 2007

74) Bell BD, Giovagnoli AR: Recent innovative studies of memory in temporal lobe epilepsy. Neuropsychol Rev 17: 455-476, 2007

75) Corbetta M, Shulman GL: Control of goal-directed and stimulus-driven attention in the brain. Nat Rev Neurosci 3: 201-215, 2002

76) Zhang Z, Lu G, Zhong Y, Tan Q, Yang Z, Liao W, Chen Z, Shi J, Liu Y: Impaired attention network in temporal lobe epilepsy: a resting FMRI study. Neurosci Lett 458: 97-101, 2009

77) Bocquillon P, Dujardin K, Betrouni N, Phalempin V, Houdayer E, Bourriez JL, Derambure P, Szurhaj $\mathrm{W}$ : Attention impairment in temporal lobe epilepsy: a neurophysiological approach via analysis of the P300 wave. Hum Brain Mapp 30: 2267-2277, 2009

78) Eisenberg DP, Berman KF: Executive function, neural circuitry, and genetic mechanisms in schizophrenia. Neuropsychopharmacology 35: 258-277, 2010

79) Boeker H, Kleiser M, Lehman D, Jaenke L, Bogerts B, Northoff G: Executive dysfunction, self, and ego pathology in schizophrenia: an exploratory study of neuropsychology and personality. Compr Psychiatry 47: 7-19, 2006

80) Wang D, Buckner RL, Fox MD, Holt DJ, Holmes AJ, Stoecklein S, Langs G, Pan R, Qian T, Li K, Baker JT, Stufflebeam SM, Wang K, Wang X, Hong B, Liu H: Parcellating cortical functional networks in individuals. Nat Neurosci 18: 1853-1860, 2015

81) Yeo BT, Krienen FM, Sepulcre J, Sabuncu MR, Lashkari D, Hollinshead M, Roffman JL, Smoller JW, Zöllei L, Polimeni JR, Fischl B, Liu H, Buckner RL: The organization of the human cerebral cortex estimated by intrinsic functional connectivity. J Neurophysiol 106: 1125-1165, 2011

Address reprint requests to: Satoshi Maesawa, MD, $\mathrm{PhD}$, Brain and Mind Research Center, Nagoya University, Department of Neurosurgery, Nagoya University Graduate School of Medicine, 65 Tsurumai, Showa, Nagoya 466-8550, Japan.

e-mail: smaesawa@med.nagoya-u.ac.jp 